\title{
Efectos de la crisis económica de 2008 sobre el empleo en las cooperativas andaluzas: un estudio del impacto provincial mediante el análisis shift-share
}

\section{Miguel Blanco Canto}

RESUMEN: Durante la reciente crisis económica, las cooperativas instaladas en la comunidad autónoma de Andalucía han demostrado ser una opción estratégica de desarrollo territorial con mayor estabilidad en el empleo frente a otras formas jurídicas empresariales. Este comportamiento es una consecuencia de su capacidad para actuar como elemento dinamizador y de adaptación a las singularidades de los territorios sobre los que actúa. Sin embargo, del análisis a nivel provincial efectuado en esta investigación se constata un comportamiento diferenciado, debido en gran medida a la existencia de diferencias socioeconómicas entre ellas.

En este trabajo se propone la utilización de la metodología shift-share para determinar sus desigualdades. Su capacidad para analizar la evolución de variables económicas en función de las características territoriales ha justificado su utilización en los análisis de desigualdad interregionales. Asimismo se ha completado el análisis con la aplicación de los modelos predictivos denominados constant share y constant shift.

Los resultados muestran diferencias sectoriales provinciales respecto al nivel de comunidad autónoma, tanto en su vertiente de cambio estructural como cambio diferencial o de competitividad regional. Asimismo, los modelos de predicción aplicados a la evolución del número de cooperativas constatan el mantenimiento de las mismas en el tiempo.

PALABRAS CLAVE: Shift-share, cooperativas, desequilibrios territoriales, empleo.

CLAVES ECONLIT: J54, P13, R10.

Cómo citar este artículo / How to cite this article: BLANCO, M. (2017): "Efectos de la crisis económica de 2008 sobre el empleo en las cooperativas andaluzas: un estudio del impacto provincial mediante el análisis shiftshare", CIRIEC-España, Revista de Economía Pública, Social y Cooperativa, 91, 175-201.

Correspondencia: Miguel Blanco Canto, Profesor del Departamento de Economía General, Universidad de Cádiz.

E-mail: miguel.blanco@uca.es. 


\section{EXPANDED ABSTRACT}

\section{Effects of the Economic Crisis of 2008 on Employment in Andaluzas Cooperatives: a Study of Provincial Impact Analysis by Shift-Share}

From the beginning of the 2008 economic crisis, several authors have suggested the need to support territorial growth initiatives by establishing incentives for local businesses. This includes incentives aimed at facilitating funding and business modernization; implementing new technologies; industrial design; improving technological quality, research and development, as well as supporting their internationalization.

Within this business network, the contribution of social economy entities to the development of territories must be stressed. On the one hand, because of its capacity to create quality jobs; on the other hand, because of its ability to overcome situations of economic crises, responding more efficiently to the changes taking place in their environments.

Thus, social economy entities have developed a rather intense activity in the process of region revitalization. The reasons to account for this fact include those connected to client proximity, their social dimension, through the prioritization of the labor factor over the capital factor, as well as their degree of efficiency in management, as a result of the labor factor's active participation. The ability of social economy entities to revitalize territories, as well as their need to adapt to the singularities of those territories, justify the need to carry out quantitative studies on their territorial impact.

This is why this paper's main goal is to analyze employment in social economy entities, by activity sectors at the provincial level in the Spanish autonomous community of Andalusia. The references to social economy entities are framed within the definition established by Article 2 of the 5/2011 Law, passed on March 29th. More precisely, they are defined as economic and business entities whose aim is the collective interest of their members, the general economic or social interest, or both at the same time. In addition, Article 5 provides the following classification: cooperatives, mutual benefit societies, foundations, associations, workforce-owned companies, social insertion companies, special employment centers, fishermen's guilds, agricultural processing companies. This classification is not closed, since other entities could be considered as long as they are ruled by the principles of people and the social good having priority over capital, applying results according to the work carried out by its members, solidary promotion at an internal level and towards society, and independence from public authorities.

Due to this, the model used is shift-share analysis, which makes it possible to connect the evolution of certain economic variables -in this research, employment in cooperatives- through the evolu- 
tion of certain characteristics associated with territories -inversion by activity sector-. This analysis is complemented by the empirical application of the constant share and constant shift estimation models, which make it possible to determine predictions, in this case, regarding employment at the provincial level in Andalusia.

The obtained results show that the negative impact on employment in cooperatives in the Andalusian autonomous community during the 2008 economic crisis has been smaller than that on businesses under other legal forms. Thereby, from 2008 to 2014, the decrease in employment in cooperatives was seven percentage points lower that the decrease in other types of companies. Breaking down this analysis by province, the obtained results show that that positive behavior has not been homogeneous in the different Andalusian provinces. Thus, the provinces of Almería and Jaén maintain the highest percentage of employees in cooperatives in relation to the total number of employees in businesses, as compared to the other provinces throughout the studied period. Applying the shift-share analyses on the evolution of employment in cooperatives at the provincial level confirms the statistical results obtained at the regional level. The provinces of Cádiz, Córdoba and Granada show negative structural changes and positive differentials. The estimation models also show that the differences among provinces are maintained.

These results may help determine the current and future existence of territories and sectors where social economy entities are underrepresented if compared to the average of the autonomous community of Andalusia and, in this case, this may allow to adapt public territorial development policies to the economic and social reality of each province.

This analysis must, in any case, be interpreted as a first approach to the study of differences in employment in cooperatives at the provincial level. Once these differences have been confirmed in this research, it is necessary to complete it by means of geographical, economic, social, environmental, demographic, and cultural variables connected to each territory. These variables are able to account for the fact that there are different territorial growth models linked to each of the studied provinces.

Launching these regional development policies requires a process of actions agreed by the different public and private agents, such as local authorities, economic agents, unions, associations, universities, etc., together with public authorities beyond the local level.

In order to be able to lead this agreed process, a unifying element is called for, to develop a strategy and to promote the actions required from all parties. Maybe the most appropriate agent to take on this role would be the local administration. The ability to revitalize territories will depend on the ability to assimilate the changes occurred in all involved territorial agents. Failing to do so, as well as taking on traditional roles, may cause a loss of opportunities, thus increasing territorial inequalities.

KEYWORDS: Shift-share, cooperatives, regional development, employment. 


\section{1.- Introducción}

En las economías actuales, las políticas de crecimiento económico constituyen la base para el incremento del nivel de renta de la ciudadanía y, de manera indirecta, contribuyen a la mejora en la consecución de determinados fines sociales como son los relacionados con el empleo, la salud, la educación o la vivienda. Por ello, el logro de determinados objetivos de crecimiento constituye un objetivo básico de toda política económica (Fernández, Parejo, y Rodríguez, 2002).

Sin embargo, tanto desde una perspectiva temporal como territorial, el crecimiento económico no es uniforme, ocasionando desequilibrios regionales persistentes debidos a la fuerte descoordinación entre las políticas económicas generales y las políticas territoriales (Alañón y Bueno, 2000). Estas situaciones han ido generando importantes desequilibrios en términos de renta per cápita o niveles de vida, que se han mantenido a lo largo del tiempo. Por ello se hace necesario el establecimiento de planes de desarrollo regional que contemplen, tanto el grado de integración entre los distintos territorios (Bendavid-Val, 2001), como el conocimiento de los recursos humanos, económicos, institucionales y culturales asociados a los mismos (Alburquerque, 2003). Desde esta perspectiva puede articularse un modelo de crecimiento territorial capaz de generar riqueza y empleo, convirtiéndose así en un soporte de relaciones sociales y actividades económicas (Rodriguez, 2009).

La fortaleza principal de este modelo de crecimiento reside en que es una estrategia que permite dar soluciones concretas a problemas específicos territoriales, usando para ello un potencial de desarrollo que no se suele utilizar en las crisis económicas, como es el estímulo de la competencia y la productividad ligada al territorio (Vázquez, 2009). Así, se puede observar un auge en la implantación de proyectos de desarrollo endógeno en etapas de crisis económicas debido a la insuficiencia de las políticas macroeconómicas para resolver los problemas de desempleo y bienestar social (Flores, 2007). En esta situación, y desde este planteamiento teórico, son los actores territoriales los que asumen el rol de aumentar la productividad en las empresas locales, así como los de mejora de la competitividad en los mercados nacionales e internacionales (Becattini, et. al., 2002), tanto a nivel empresarial como territorial. Desde la perspectiva de la empresa, sus gestores pueden conseguir el incremento de su capacidad para suministrar bienes y servicios de una manera más eficaz y eficiente, lo que implica el desarrollo de habilidades que les permitan obtener utilidades que se manifiesten en reinversión 1 (Labarca, 2007). Desde los territorios, los actores sociales pueden sostener y expandir su nivel de participación en otros mercados territoriales a la vez que logran elevar el nivel de vida de la ciudadanía (Sobrino, 2005).

1.- La capacidad de mejora de la eficiencia empresarial puede conseguirse mediante la combinación de los siguientes cinco elementos: Dirección y gestión empresarial; innovación productiva; coordinación durante los procesos de producción; organización industrial; política industrial activa. 
Desde este enfoque, el desarrollo no se sustenta de manera exclusiva en aspectos puramente económicos, ya que incorpora otros elementos para dar una solución eficiente y global. Así, el desarrollo endógeno se apoya en dos elementos básicos, la identidad cultural y la participación de los entes locales. El territorio pasa a convertirse en un espacio social donde la solidaridad entre los actores ligados al mismo conduce a un compromiso social de pertenencia a un lugar geográfico determinado y, en el cual, desarrollan sus actividades (Ramos y Garrido, 2011). Esta dinámica hace que se creen cuatro dimensiones profundamente interconectadas: desarrollo social y humano (acceso y mejora de la educación, mejora de la distribución del ingreso para la inclusión social, desarrollo de formas de economía social y solidaria,...); desarrollo "sustentable" (puesta en valor del patrimonio natural y cultural, fomento de las energías renovables, consumo responsable, ...); desarrollo institucional y cultural (creación de redes y capital social territorial); desarrollo económico (fomento de la diversificación y la calidad productiva, sistema fiscal y marco jurídico adecuado al fomento de las empresas locales,...) (Alburquerque, 2004).

Por todas estas implicaciones, desde la crisis económica de 2008 , son varios los autores que han planteado un enfoque territorial como respuesta a los desequilibrios regionales que se están originando, fuera de medidas estrictamente macroeconómicas (Casalis, 2008; Capel, 2009; Pérez y Pizarro; 2012). En la práctica, los principales problemas para el desarrollo de estas políticas de crecimiento endógeno son los derivados de la creación y mantenimiento de empresas, la falta de adecuación de la mano de obra a los puestos ofertados o la inexistencia de un ente integrador. De ellos, el factor empresarial constituye el elemento clave para el crecimiento local (Vázquez, 2009). La capacidad empresarial determina así la competitividad y el crecimiento económico de un territorio (García, Melián, 2003). Igualmente impulsa la regeneración del tejido productivo territorial en un momento de gran competencia entre empresas, debido, entre otros factores, al proceso de globalización (De Paz, 2008), que ha creado nuevas vías de interacción, como son el auge del comercio internacional, las inversiones directas extranjeras, la integración financiera, la globalización de la información, del know-how o las nuevas tecnologías de las comunicaciones (Vázquez, 2009).

Este tejido productivo local está básicamente constituido por pequeñas y medianas empresas que requieren medidas específicas de apoyo que les permitan mejorar su productividad y competitividad. Entre ellas se encuentran actuaciones dirigidas a facilitar su financiación, a la modernización empresarial, implantación de nuevas tecnologías, diseño industrial, mejora de la calidad, la investigación y desarrollo tecnológico, así como el apoyo a la internacionalización de su actividad.

Dentro de este entramado empresarial, resulta destacable la aportación que han venido ofreciendo las entidades de economía social al desarrollo de los territorios. Por un lado, por su capacidad de generar empleo de calidad. Por otro, por su capacidad de superar situaciones de crisis económicas, al responder de una manera más eficiente a los cambios que se vienen produciendo en su entorno (Pérez y Valiente, 2015; Fuentes y Mainar, 2015). De esta manera, las entidades de economía social han ido desarrollando una actividad muy intensa en el proceso de revitalización de las regiones (Pérez y Pizarro, 
2012). Tarazona y Albors (2005), enumeran, como elementos de conexión entre las Entidades de Economía Social y el desarrollo territorial, las relacionadas con la proximidad a los clientes, su dimensión social mediante la priorización del factor trabajo sobre el factor capital, y su grado de eficiencia en la gestión como consecuencia de la participación activa del factor trabajo en la gestión. Esta capacidad que tienen las entidades de economía social para dinamizar los territorios, y su necesidad para adaptarse a las singularidades de los mismos, justifican la necesidad de realizar estudios cuantitativos de impacto territorial.

En este artículo, las referencias a las entidades de economía social se encuadran dentro de la definición que hace el legislador en el artículo 2 de la ley 5/2011 de 29 de marzo. Concretamente las define como aquellas entidades económicas y empresariales que persiguen el interés colectivo de sus integrantes, el interés general económico o social 0 ambos a la vez. Asimismo, en su artículo 5 las clasifica en cooperativas, mutualidades, fundaciones, asociaciones, sociedades laborales, empresas de inserción, centros especiales de empleo, cofradías de pescadores, sociedades agrarias de transformación. Esta clasificación no es cerrada, pudiendo tener dicha consideración las entidades que se rijan por los principios de primacía de las personas y el bien social sobre el capital, aplicación de resultados en función del trabajo realizado por sus integrantes, promoción solidaria interna y con la sociedad e independencia de los poderes públicos².

Esta creciente importancia de desarrollo regional -tanto a nivel teórico como práctico- mediante el apoyo a las entidades de economía social, y su incorporación a la agenda de política europea, nacional y regional, justifican la existencia de investigaciones sobre el nivel de impacto en los territorios. Por esa razón, en este artículo se fija como objetivo principal el análisis del empleo en las cooperativas, por sectores de actividad a nivel provincial en la comunidad autónoma de Andalucía. Para ello, el modelo utilizado es el análisis shift-share, que permite relacionar la evolución de determinadas variables económicas -en este trabajo de investigación, el empleo en las cooperativas- mediante la evolución de determinadas características asociadas a los territorios -inversión por sectores de actividadEste análisis se complementa con la aplicación empírica de los modelos de previsión constant share y constant shift que permiten determinar predicciones, en este caso, sobre el empleo provincial de las provincias andaluzas.

Los resultados obtenidos indican desigualdades territoriales en el empleo asociado a las entidades de economía social que se mantendrán en el tiempo. Así, esta investigación puede considerarse como un análisis exploratorio que posteriormente será objeto de una investigación más amplia para determinar las causas de estos desequilibrios. Los resultados obtenidos pueden servir para determinar la existencia actual y futura de territorios y sectores donde las entidades de economía social se

2.- Son también destacables las definiciones ofrecidas por el comité nacional de liaison des activités coopératives, mutuelles et associatives (CNLAMCA) que publica en 1980 la charte de l'économie sociale, el conseil Wallon de l'économie sociale (1990), la conferencia europea permanente de cooperativas, mutualidades, asociaciones y fundaciones (CEP-CMAF), la comisión científica del CIRIEC-España (1990) o los documentos elaborados para la comisión europea por Barea y Monzón (2006). 
encuentran infrarrepresentadas en relación a la media de la comunidad autónoma de Andalucía y, en este caso, puede permitir adecuar políticas públicas de desarrollo territorial a la realidad económico y social de cada una de ellas.

La estructura que recoge el artículo se presenta a continuación: tras este epígrafe introductorio, donde se justifica esta investigación y se establece un marco teórico que pone de manifiesto la importancia de la economía social en la dinamización y desarrollo de los territorios, se analiza en el apartado segundo la actuación de las administraciones públicas para facilitar su implantación y desarrollo. El tercer apartado analiza la evolución del empleo en las cooperativas andaluzas durante la crisis económica de 2008; En los epígrafes 4 y 5 se presentan la aplicación metodológica propuesta, basada en un análisis shift-share y los modelos de predicción utilizados. En el sexto y séptimo epígrafe se recoge la aplicación empírica cuyos resultados son analizados, para finalizar con las conclusiones y una propuesta de debate.

\section{2.- Las administraciones públicas y las entidades de la economía social}

Desde las distintas administraciones públicas -europeas, nacionales y regionales-, conscientes del potencial de generación de riqueza y empleo por parte de los territorios, se han ido adaptando estas teorías hacia planteamientos político-administrativos de desarrollo local. De esta manera se reconoce el protagonismo de las entidades de economía social en los procesos de revitalización de los territorios y, como consecuencia de ello, se han ido aprobando diversas normativas y programas de apoyo a las mismas. De esta manera, si se logra establecer una dinámica de cooperación e innovación que permita a estos actores territoriales aprovechar los recursos específicos y el capital social, el territorio pasará a tener la consideración de activo estratégico esencial (Alburquerque, 2004: Ramos y Garrido, 2011).

Así, se ha ido incentivado la creación de empresas participadas por trabajadores mediante la fórmula de emprendimiento. Este nuevo empresariado es concebido como un elemento de dinamización de los mercados de factores y los mercados de productos. Su aparición puede ser el resultado de la tradición empresarial, o la existencia de una situación crítica del territorio que obligan a la ciudadanía a la emigración o al autoempleo (Rodríguez, 2009). En cualquier caso, el estímulo a la capacidad empresarial local es la consecuencia de la creación de una cultura emprendedora territorial, por medio de un ambiente favorable hacia la figura del emprendedor que disminuya la falta de motivación y facilite la generación de nuevas propuestas empresariales adaptadas a las características regionales. Esta iniciativa emprendedora es uno de los principales impulsores de la innovación, la competitividad y el desarrollo económico y social (Carree y Thurik, 2003) y como tal, deberían de apoyarse. 
En concreto, la Unión Europea ha ido adaptando las teorías de desarrollo local hacia planteamientos político-administrativos mediante la aprobación de diferentes normativas y proyectos. En 1989 se presenta una comunicación de la comisión al consejo titulada "Las empresas de la economía social y la realización del mercado europeo sin fronteras". Desde entonces, destacar el reglamento por el que se aprueba el estatuto de la sociedad cooperativa europea (Reglamento CE 1435/2003), la directiva 2003/72/CE del consejo de 22 de julio por la que se completa el estatuto de las sociedades de economía social, el "informe del parlamento europeo sobre economía social" de 26 de enero de 2009, la Comunicación de la Comisión sobre la Iniciativa en favor del emprendimiento social (Social Bussines Initiative)(COM(2011)0682), la Resolución del Parlamento Europeo, de 2 de julio de 2013, sobre la Contribución de las cooperativas a la salida de la crisis (2012/2321(INI)) o la comunicación de la Comisión al Consejo, al Parlamento Europeo, al Comité Económico y Social Europeo y al Comité de las Regiones sobre la revisión de la «Small Business Act» para Europa (COM(2011) 78 final).

Esta tendencia europea de reconocimiento y apoyo a la economía social se ha transmitido a los países de la Unión Europea. Destacar la incorporación efectuada a su ordenamiento jurídico interno de Francia (Ley de 20 de julio n $\mathrm{n}^{\circ}$ 83-657 relativa al desarrollo de ciertas actividades de economía social, Ley de 12 de julio de $1985 n^{\circ} 85-703$ y más actualmente la Ley de 21 de julio de $2014 n^{\circ} 387$ relativa a la Economía Social y Solidaria) o Bélgica (Decreto relativo a la economía social de la región de Valonia, de 20 de noviembre de 2008).

En España se publica la ley 5/2011 de 29 de marzo sobre economía social, donde se establece su ámbito de aplicación, sin perjuicio de las competencias que pueda corresponder a las comunidades autónomas que la hayan asumido en sus estatutos de autonomía. En la comunidad autónoma de Andalucía, al amparo de lo establecido en los artículos 58, 157, 163 y 172 del estatuto de autonomía para Andalucía aprobado mediante la ley Orgánica 2/2007 de 19 de marzo se aprueba el decretoley 8/2013 o el III pacto andaluz por la economía social.

El decreto-ley 8/2013 de 28 de mayo de medidas de creación de empleo y fomento al emprendimiento desarrolla en su título IV un programa de apoyo a la economía social de fomento del empleo estable, cualificado y de calidad, así como de apoyo a los proyectos innovadores y que favorezcan la competitividad de las cooperativas y de las sociedades laborales mediante tres líneas subvencionables:

- Fomento del empleo en cooperativas y sociedades laborales

- Fomento del emprendimiento social

- Intercooperación empresarial en la economía social.

A nivel regional, en la comunidad autónoma de Andalucía se aprueba el 16 de diciembre de 2011 el III pacto andaluz por la economía social, en el que se plantea la potencialidad de las cooperativas y las sociedades laborales en el desarrollo de los territorios, en un contexto como el actual de crisis económica mediante el apoyo de las iniciativas de los emprendedores hacia entidades que se creen al amparo de este tipo de forma jurídica. 
Para ello se desarrolla mediante las siguientes fuentes:

- Principios y valores de la economía social

- Actividad económica y desarrollo empresarial

- Empleo de calidad y relaciones laborales

- Desarrollo local y rural, como instrumento para la cohesión social

- Vertebración y organización de la economía social andaluza

- Desarrollo, seguimiento y evaluación del pacto

Destacar como ejemplo de cooperación entre las distintas administraciones públicas europeas, nacionales y regionales, el proyecto PASE (políticas públicas y empresas de economía social) desarrollado entre 2008 y 2011 y financiado conjuntamente por el programa Interreg IVC y el fondo de desarrollo regional europeo. Los socios del proyecto fueron la Junta de Andalucía y el Consorcio MadridSur; la ciudad de Forest en la región de Bruselas (Bélgica); el consejo regional Provence-Alpes-Côte d'Azur (Francia); el centro de educación de adultos de la ciudad de Hannover (Alemania); la unidad laboral, de educación y formación de la región Marche (Italia); la Universidad de economía de Cracovia (Polonia); y el gobierno regional de Arges (Rumanía).

Su objetivo principal durante el período de ejecución ha sido el refuerzo de la efectividad de las políticas públicas regionales en la promoción y el apoyo al emprendimiento social como un criterio para el desarrollo local y la competitividad territorial.

En él se contemplan cuatro líneas de trabajo: Identificación, selección y comparación de buenas prácticas concernientes a las políticas públicas de apoyo a las empresas sociales; Profundización en el conocimiento de las buenas prácticas seleccionadas para facilitar su transferencia en los países receptores; transferencia de buenas prácticas a los países receptores, sobre la base de una hoja de ruta definida por todos los socios; desarrollo de guías o pautas conjuntas para mejorar la capacidad de las autoridades públicas locales de gestionar las contrataciones públicas para la provisión de servicios de interés general a través o mediante empresas sociales. 
3.- Evolución del empleo en las cooperativas andaluzas durante la crisis económica de 2008

Según la información suministrada por la base de datos de economía social del Ministerio de Empleo y Seguridad Social, en 2015 el número de entidades de economía social ascendía en España a 42.9293. De ellas, el 24\% estaban en la comunidad autónoma de Andalucía, y el 47\% (20.258) eran cooperativas. Por la importancia relativa de estas cifras, el análisis presentado en este artículo está centrado en las cooperativas que desarrollan su actividad en la comunidad autónoma de Andalucía.

En el año 2008, de las 2.361 .398 personas que trabajaban en alguna de las empresas de Andalucía, el número de ocupados en cooperativas ascendía a 50.984. En 2014, el número de trabajadores andaluces se reduce a 1.844.753, lo que supone una disminución del 22\%. Para las empresas cooperativas, la disminución fue del 15\% contabilizándose 43.085 personas que desarrollaban su actividad laboral en el año 2014.

La Figura 1 recoge la evolución porcentual del número de empleados en cooperativas sobre el total de empleados en empresas durante el período 2008-2014. En él se aprecia una mayor estabilidad en el empleo para los trabajadores en cooperativas frente a los que desarrollaron su trabajo en empresas pertenecientes a otros tipos de personalidad jurídica.

\section{Figura 1. Porcentaje de empleados en cooperativas sobre el total de empresas}

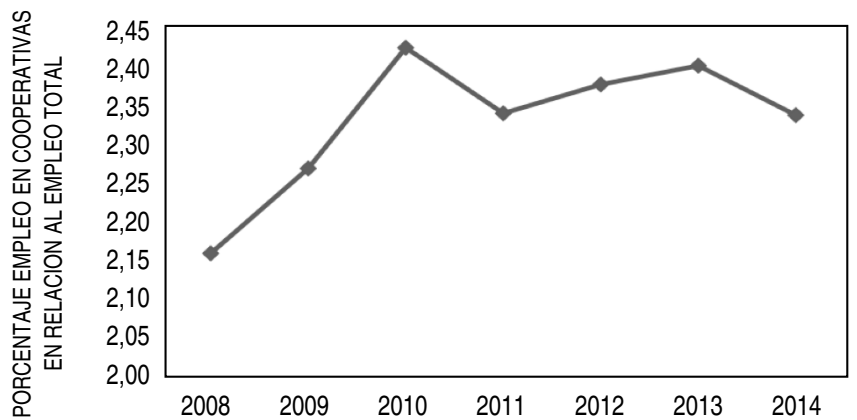

FUENTE: base de datos de la economía social del Ministerio de Empleo y Seguridad Social (fecha de consulta: $28 / 03 / 2016)^{4}$ 
En la figura 2 se ha desagregado el número de empleos totales según la forma jurídica de la empresa. En él se puede apreciar como las sociedades cooperativas han mostrado mayor estabilidad durante la crisis económica de 2008 que otros tipos de empresas.

\section{Figura 2. Número de empleados en empresas según forma jurídica}

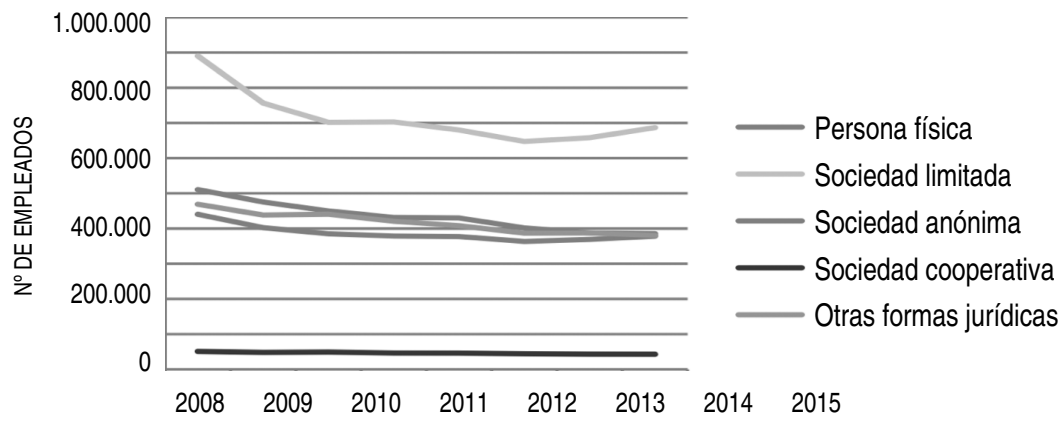

FUENTE: Base de datos de la economía social del Ministerio de Empleo y Seguridad Social (fecha de consulta: 28/03/2016).

De esta manera se pone de manifiesto, para un contexto de crisis económica como el actual, el efecto positivo para el empleo realizado por este tipo de entidades de economía social, consolidándose como una alternativa menos sensible a las perturbaciones cíclicas de la economía.

En este comportamiento ha sido decisiva la primacía del aspecto social sobre el capital. En caso de disminución de los beneficios o existencia de pérdidas, es más probable el reparto de responsabilidades entre los socios, asumiendo cada uno de ellos un descuento en sus retribuciones antes de recurrir a la salida de la empresa. En el resto de empresas, por parte del empresariado no cooperativo, la primacía del capital y la necesidad de dar cuentas a los accionistas o participantes del capital puede llevar con mayor facilidad al despido de trabajadores para disminuir costes.

Otros factores analizados por los investigadores para explicar la menor sensibilidad a las recesiones se encuentran en las investigaciones de Clemente, et. al, (2014), Cuadrado y Ciruela, (2014) 0 Salinas y Osorio, (2012); su capacidad de innovación y su intensidad exportadora (Clemente, et. al, 2014); la proximidad a los mercados y su mejor adaptación a las necesidades de sus socios y clientes (Cuadrado y Ciruela, 2014); las nuevas formas de organización del trabajo, el compromiso con la sociedad y la adaptación a las necesidades de inserción sociolaboral de los colectivos con especiales dificultades de acceso al mercado (Salinas y Osorio, 2012) 
Con respecto a las investigaciones sobre el carácter anticíclico en la evolución del empleo en cooperativas y otras entidades de economía social durante las crisis económicas, destacar las aportaciones de Monzón (1991) o Grávalos y Pomares (2001). En concreto, Grávalos y Pomares analizan la evolución del empleo en cooperativas a nivel regional durante la crisis del 1992, llegando a determinar el carácter anticíclico en la evolución del empleo en cooperativas. Justifican este comportamiento como consecuencia de las dinámicas de transformación encaminadas a mantener los puestos de trabajo en momentos de incertidumbre. Asimismo destacan como los desempleados pertenecientes a colectivos con especiales dificultades para acceder a un puesto de trabajo buscan en la economía social un camino de entrada en el mercado laboral.

Recientemente, y centrándose en los efectos de la crisis económica de 2008, destacar las aportaciones de Izquierdo y Vicedo (2009), Navarro y Climent (2010), Díaz y Marcuello (2010), Encinas, et. al. (2011) o Ramos (2012). Encinas, et. al (2011) considera que esta situación puede explicarse por la capacidad de estas organizaciones para re-inventarse y adaptarse de una manera rápida a los nuevos requerimientos del entorno y, de esta manera, seguir formando parte del tejido empresarial local. Para Melián (2010), el mejor comportamiento del empleo es consecuencia de la propia filosofía de desarrollo de las entidades sociales al propiciar una mejor respuesta a las etapas de crisis que las entidades capitalistas. Su nacimiento como consecuencia del esfuerzo de los emprendedores sensibilizados con una problemática territorial concreta, la creación de empleo asociada a un territorio determinado, y la no deslocalización de su sistema productivo podrían justificar esta situación.

La tabla 1 recoge la evolución del empleo provincial en sociedades cooperativas y total de empresas para el período 2008-2014. Un primer análisis de los datos ofrecidos indica una distribución territorial desigual a nivel interprovincial. Así, la provincia que mayor número de empleos mantiene en empresas que han optado por la forma jurídica de cooperativas es Almería (30\%), seguida de Sevilla (21\%). Ambas concentran el $51 \%$ del empleo. Granada ocupa el tercer puesto (13\%), seguido de Jaén (11\%), Córdoba (9\%), Málaga (7\%), Cádiz (5\%) y Huelva (4\%). 


\section{Tabla 1. Empleo provincial en Sociedades cooperativas y total de empresas. Período 2008-2014}

\begin{tabular}{|c|c|c|c|c|c|c|c|c|c|c|c|}
\hline \multirow[b]{2}{*}{ Años } & & \multicolumn{10}{|c|}{ Número de empleados } \\
\hline & & Almería & Cádiz & Córdoba & Granada & Huelva & Jaén & Málaga & Sevilla & $\begin{array}{r}\text { Resto de } \\
\text { España }\end{array}$ & TOTAL \\
\hline \multirow[t]{2}{*}{2008} & Sociedad & 14701 & 3106 & 4545 & 6359 & 2328 & 5583 & 4339 & 9481 & 542 & 50984 \\
\hline & Total & 164157 & 232930 & 168036 & 197133 & 93692 & 119174 & 381336 & 668952 & 335988 & 2361398 \\
\hline \multirow[t]{2}{*}{2009} & Sociedad coop. & 13636 & 2587 & 4297 & 6092 & 2171 & 5281 & 3897 & 9714 & 408 & 48083 \\
\hline & Total & 140416 & 194098 & 146738 & 169356 & 79903 & 103422 & 331828 & 615565 & 339214 & 2120540 \\
\hline \multirow[t]{2}{*}{2010} & Sociedac & 14802 & 2379 & 4205 & 6320 & 2102 & 4797 & 3617 & 10306 & 541 & 49069 \\
\hline & Total & 134429 & 182821 & 137999 & 159455 & 75717 & 97102 & 306751 & 602760 & 329193 & 2026227 \\
\hline \multirow[t]{2}{*}{2011} & Sociedad coop. & 12966 & 1892 & 4099 & 6016 & 1992 & 5078 & 3043 & 10760 & 445 & 46291 \\
\hline & Total & 126607 & 178173 & 134853 & 157284 & 71305 & 93672 & 292935 & 594857 & 330347 & 1980033 \\
\hline \multirow[t]{2}{*}{2012} & Sociedad coop. & 13817 & 1787 & 3972 & 6098 & 1713 & 5223 & 3177 & 9728 & 580 & 46095 \\
\hline & Total & 123560 & 174050 & 130601 & 150045 & 68288 & 89241 & 284447 & 584637 & 336205 & 1941074 \\
\hline \multirow[t]{2}{*}{2013} & Sociedad coop. & 13725 & 1534 & 3749 & 5761 & 1691 & 4283 & 3141 & 9888 & 440 & 44212 \\
\hline & Total & 120244 & 163671 & 120031 & 142928 & 64840 & 83229 & 277735 & 563005 & 307489 & 1843172 \\
\hline \multirow[t]{2}{*}{2014} & Sociedad coop. & 13628 & 1608 & 3578 & 5841 & 1704 & 4781 & 3012 & 8469 & 464 & 43085 \\
\hline & Total & 122795 & 159393 & 119881 & 143408 & 64464 & 84327 & 283310 & 555603 & 311572 & 1844753 \\
\hline
\end{tabular}

FUENTE: Base de datos de la economía social del Ministerio de Empleo y Seguridad Social (fecha de consulta: 28/03/2016).

Se ha elaborado la figura 3 para mostrar la evolución experimentada por el porcentaje de empleados en cooperativas sobre el empleo total de empresas. Como se puede apreciar, el comportamiento mostrado a nivel provincial es muy heterogéneo. Granada experimenta un incremento del $26 \%$ en el número de empleados en el período 2008 a 2014. Le sigue Almería con un 23\% y Jaén con un $21 \%$. Córdoba y Sevilla presentan unos incrementos más modestos (10\% y $7 \%$ respectivamente). Cádiz y Málaga son provincias que representan una evolución negativa, concretamente del $-24 \%$ y $-6 \%$ respectivamente. 


\section{Figura 3. Evolución provincial del porcentaje de empleados en cooperativas sobre el empleo total de empresas}

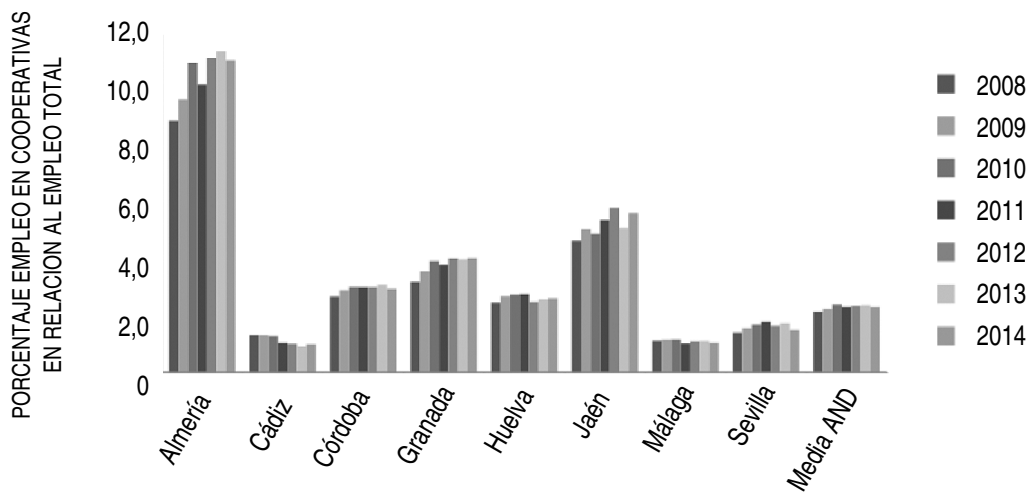

FUENTE: Base de datos de la economía social del Ministerio de Empleo y Seguridad Social (fecha de consulta: 28/03/2016).

Destacar la evolución provincial positiva en el porcentaje de empleados en cooperativas sobre el empleo total de empresas que muestran las provincias de Almería y Jaén. Esta situación probablemente pueda explicarse por el mayor arraigo cultural cooperativista asociado a dichos territorios (Cuadrado y Ciruela, 2014).

Todos estos datos evidencian como en Andalucía existen diferencias importantes interprovinciales a nivel regional relacionadas con el número de empresas cooperativas y el empleo generado mediante ellas. Con carácter general, estas diferencias pueden ser explicadas como el resultado de una serie de contrastes territoriales de carácter socioeconómicos que se mantienen en el tiempo, especialmente relacionados con el grado de competitividad empresarial asociado a núcleos de desarrollo muy específicos (Pita y Pedregar, 2015).

En todo caso, el reconocimiento de estas singularidades asociadas a cada provincia hace necesario profundizar en los análisis territoriales y, en base a ellos, definir políticas de desarrollo adecuadas a las características endógenas de cada uno de los territorios. Estos análisis pueden apoyarse en técnicas cuantitativas de disparidad regional mediante las cuales puedan elaborarse cuadros de resultados que evidencien situaciones de disparidad, como medidas de dispersión regional, coeficientes de asociación geográfica de Florence, coeficiente T de Theil, cocientes de localización regional, sectorial, diversificación o de especialización. De todas las técnicas utilizadas, la que ha alcanzado mayor grado de difusión en los análisis de disparidades regionales es el análisis shift-share. Esta técnica permite asimismo, incorporar modelos de predicción. De esta manera se pueden estimar desequilibrios 
territoriales y determinar si estos se mantienen en el tiempo. Los resultados obtenidos pueden constituir la base de nuevas investigaciones que determinen los factores que mantienen, incrementan 0 disminuyen la variabilidad interregional.

\section{4.- El análisis shift-share}

El análisis shift-share es una técnica estadística tradicionalmente utilizada por los investigadores para analizar diferencias regionales, tanto a nivel nacional como a nivel internacional. A nivel internacional destacar los trabajos de Timmer y Szirmai (2000) sobre el incremento de la productividad en el sector manufacturero durante el período 1963-1996 en cuatro países asiáticos; López y Mayor (2002) y la incidencia de los factores sectoriales -nacionales, industriales y competitivos- en los desequilibrios regionales o Patuelli, Reggiani, Nijkamp y Blien (2006) que analizan la evolución sobre el empleo en 439 distritos alemanes.

En España, destacar los trabajos realizados por Villaverde (1996) sobre la evolución regional de la producción y la productividad del empleo en el período 1980-1993; Mayor, López y Pérez (2005) aplican el análisis shift-share para identificar los distintos componentes nacionales, sectoriales y competitivos que han originado la evolución del empleo en el período 2001-2003, así como la realización de un conjunto de predicciones sobre el comportamiento de variables asociadas al empleo por sectores; Ramajo y Márquez (2008) lo aplican al sector manufacturero de bienes de equipo en las regiones penisulares españolas durante el período 1970-2004.

En este artículo se ha utilizado el análisis para determinar el impacto provincial del empleo en cooperativas en la Comunidad Autónoma de Andalucía.

El análisis shift-share es una técnica basada en la descomposición por partes (share) de las variaciones (shifts) experimentadas en un período relativas a varias unidades territoriales (Martín-Guzman, Martín, 1987). Mediante esta metodología se incorporan las características territoriales en la evolución de variables económicas.

La variable utilizada en este artículo es el empleo en cooperativas y la unidad territorial de referencia es la Comunidad Autónoma de Andalucía, dividida por provincias. 
Se parte de la siguiente igualdad:

$$
C_{I J}=n_{i j}+p_{i j}+d_{i j}
$$

$C_{i j}=$ Variaciones en el empleo en cooperativas durante un período de tiempo definido referida a un sector de actividad i en la provincia j

$n_{i j}=$ Componente territorial a nivel de la Comunidad Autónoma de Andalucía para los sectores de actividad y provincias

$p_{i j}=$ Desplazamiento estructural

$\mathrm{d}_{\mathrm{ij}}=$ Desplazamiento diferencial

Y se definen las variaciones experimentadas entre el período 1 y el período 2 mediante la definición de las siguientes tasas de variación,

$r=$ Tasa de variación en el número de empleados en las cooperativas a nivel autonómico para todo el conjunto de sectores de actividad

$r_{i}=$ Tasa de variación en el número de empleados en las cooperativas relativas al sector de actividad i-esimo.

$r_{i j}=$ Tasa de variación en el número de empleados en las cooperativas de un sector de actividad determinado relativa a la provincia $j$.

La expresión (1) queda convertida en,

$$
C_{\mid J}=V_{i j} r_{i j}
$$

Agregando los diferentes sectores de actividad,

$$
\Sigma C_{l J}=\sum V_{i j} r+\sum V_{i j}\left(r_{i}-r\right)+\sum V_{i j}\left(r_{i j}-r_{i}\right)
$$

que puede expresarse

$$
r_{j}-r=\sum\left(S_{i j}-S_{i}\right) r i+\sum S_{i j}\left(r_{i j}-r\right)
$$

donde

$r_{j}-r$ es el cambio neto

El cambio neto indica la variación de cada provincia respecto a la experimentada en la Comunidad Autónoma de Andalucía. Si el sector a nivel de provincia experimenta un crecimiento por encima del desarrollado a nivel de la Comunidad Autónoma, el cambio neto obtenido ofrece valores positivos. 
El cambio neto se ha descompuesto en dos cambios: cambio estructural y cambio diferencial.

$\sum\left(S_{i j}-S_{j}\right)$ ri es el cambio estructural

Este cambio estructural muestra los efectos de la estructura productiva provincial como la diferencia entre cada uno sus sectores y el análogo autonómico. Si $r_{i}$ es mayor que $r$, indica una evolución positiva provincial. En el caso contrario, la evolución de la provincia es inferior a la evolución autonómica.

$\Sigma \mathrm{S}_{\mathrm{ij}}\left(\mathrm{r}_{\mathrm{ij}}-\mathrm{r}\right)$ es el cambio diferencial

El cambio diferencial indica las diferencias sectoriales de cada provincia con respecto a la variación experimentada a nivel de la comunidad. Si $r_{i j}$ es mayor que $r_{j}$ indica un crecimiento más rápido a nivel provincial que regional.

\section{5.- Modelos de predicción}

Las limitaciones del modelo descritas por autores como Stillwell (1969) o Bartels, Nicol y Van Duijin (1982) han llevado a los investigadores a la utilización de esta técnica con carácter meramente descriptivo (Martín-Guzman, Martín, 1987). Para el desarrollo de un modelo predictivo, se han desarrollado otras técnicas como los denominados constant share y constant shift.

El modelo de predicción constant share propuesto por Hewings (1976) se basa en la aplicación a Ios diferentes sectores regionales de las variaciones previstas a nivel nacional (James y Hughes, 1973, Brown, 1969)). De esta manera, se da un tratamiento homogéneo territorial al crecimiento sin ningún tipo de diferenciación regional.

Los modelos de predicción se han aplicado sobre el número de empresas cooperativas en la comunidad autónoma de Andalucía a nivel provincial. La variación en el número de cooperativas de un sector i experimentado por una región j sería el resultado de aplicar la ecuación

$$
E_{i j}^{t+1}=\left(1+r_{j}\right) E_{i j}^{t}
$$

Donde $E_{i j}^{t+1}$ sería la previsión en el número de empresas cooperativas por sectores del año $t+1$ en el sector i para la región j. En esta investigación se ha calculado para el período 2008 a 2015 en la región: Andalucía. 
$r_{i}$ sería la tasa de crecimiento nacional correspondiente a ese año (prevista). Los datos utilizados son los publicados por el banco de España mediante sus informes de proyecciones de la economía española desde 2008 a 2015.

Finalmente, $E_{i j}^{t}$ son los datos de cooperativas por sectores del año t.

El modelo constant shift considera que el deferencial de cooperativas regionales se mantiene en una comparativa temporal. Así su número previsto para el período $t+1$ se calcula mediante la expresión

$$
E_{i j}^{t+1}=\left(1+r_{i}^{t+1}+s_{i}^{t+1}\right) E_{i j}^{t}
$$

donde

$$
s_{i}^{t+1}=r_{i j}^{t+1}-r_{j}^{t+1}
$$

En este artículo, para el cálculo de $r_{i j}$ y $r_{j}$ se han tenido en cuenta los valores históricos de los niveles de empresas cooperativas referenciados a los sectores definidos en la Comunidad Autónoma de Andalucía según los datos proporcionados por el Instituto de Estadística y Cartografía de Andalucía.

Una vez estimadas las previsiones se han calculado los errores a posteriori correspondiente a los años 2009 a 2014 a fin de determinar los modelos que expresan de manera más fiable la evolución de las series temporales. En concreto, se ha calculado la suma de errores porcentajes de error absoluto, el porcentaje medio del error absoluto, la raíz del error cuadrático medio y el índice de Theil.

\section{6.- Análisis Shift-Share aplicado a las cooperativas}

La figuras 4 muestran los resultados del análisis Shift-Share aplicado a la evolución del empleo en las cooperativas -a nivel sectorial- de la Comunidad Autónoma de Andalucía para el período 20082014. Los datos se han obtenido mediante la información suministrada por el directorio de empresas y establecimientos con actividad económica en Andalucia ${ }^{5}$. En él se recoge la posición relativa de cada provincia en función de dos puntos. El primero hace referencia al cambio estructural y el segundo al cambio diferencial.

5.- Según datos publicado por el Instituto de Estadística de Andalucía (http://www.juntadeandalucia.es/institutodeestadisticaycartografia/iea/consultasActividad.jsp?CodOper=37\&sub=61671). 


\section{Figura 4. Análisis Shift-Share. Cambio estructural y cambio diferencial}

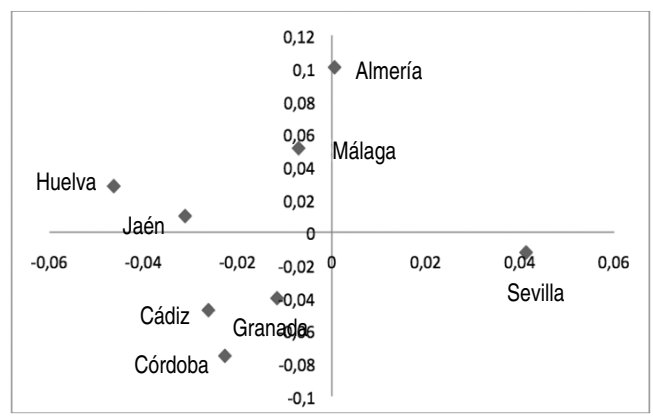

FUENTE: Base de datos de la economía social del Ministerio de Empleo y Seguridad Social (fecha de consulta: 28/03/2016).

Como se puede apreciar, en las provincias de Cádiz, Córdoba y Granada la evolución que presenta su estructura productiva por sectores es inferior al experimentado por la comunidad autónoma de Andalucía. Al ser también su cambio diferencial negativo, sus diferencias sectoriales con respecto a la variación experimentada por la comunidad son inferiores.

En el caso de Huelva, Jaén y Málaga mantienen durante el período seleccionado cambios estructurales negativos. Sin embargo, al presentar cambios diferenciales positivos, el crecimiento sectorial recogido es mayor al de la región autonómica. Situación totalmente contraria es la experimentada por la provincia de Sevilla que mantiene cambios positivos estructurales y negativos en el componente competitivo.

Almería es la única provincia de Andalucía que mantiene ambos componentes con valores positivos.

Estos datos ponen de manifiesto una distribución territorial desigual entre las provincias de la comunidad autónoma de Andalucía. Especialmente significativo es el caso de Cádiz, Córdoba y Granada, que mantienen tanto una representación por sectores inferior a la media, como una evolución temporal menor. 


\section{7.- Aplicación de modelos de previsión a las cooperativas andaluzas}

Los resultados de la aplicación de los modelos de previsión y los errores asociados a cada uno de ellos aparece en las siguientes tablas. Concretamente, las tablas 2 y 3 recogen la evolución en el número de cooperativas mediante la aplicación del modelo de predicción constant-share y sus errores asociados. Las tablas 4 y 5 , la evolución en el número de cooperativas según el modelo de predicción constant shift y sus errores.

\section{Tabla 2. Evolución del número de Cooperativas. Modelo de predicción constant share}

\begin{tabular}{|l|c|c|c|c|c|c|c|c|c|}
\hline & $\begin{array}{c}\text { Industria, energía, } \\
\text { agua y gestión } \\
\text { de residuos }\end{array}$ & Construcción & Comercio & $\begin{array}{c}\text { Transporte } \\
\text { y almacena- } \\
\text { miento }\end{array}$ & Hostelería & $\begin{array}{c}\text { Información } \\
\text { y comunica- } \\
\text { ciones }\end{array}$ & $\begin{array}{c}\text { Banca } \\
y \\
\text { seguros }\end{array}$ & $\begin{array}{c}\text { Servicios } \\
\text { sanitarios, } \\
\text { educativos } \\
\text { y resto servicios }\end{array}$ & Total $^{*}$ \\
\hline $\mathbf{2 0 0 9}$ & 1764 & 859 & 1488 & 272 & 265 & 70 & 31 & 1060 & 5904 \\
$\mathbf{2 0 1 0}$ & 1653 & 731 & 1341 & 238 & 233 & 61 & 27 & 1031 & 5273 \\
$\mathbf{2 0 1 1}$ & 1519 & 650 & 1257 & 236 & 211 & 55 & 24 & 1006 & 4988 \\
$\mathbf{2 0 1 2}$ & 1421 & 612 & 1202 & 239 & 190 & 56 & 21 & 1009 & 4806 \\
$\mathbf{2 0 1 3}$ & 1271 & 543 & 1156 & 223 & 178 & 49 & 23 & 964 & 4414 \\
$\mathbf{2 0 1 4}$ & 1259 & 531 & 1177 & 215 & 180 & 48 & 23 & 977 & 4431 \\
$\mathbf{2 0 1 5}$ & 1236 & 490 & 1170 & 223 & 175 & 48 & 23 & 1000 & 4434 \\
$\mathbf{2 0 1 6}$ & 1247 & 492 & 1178 & 224 & 175 & 48 & 23 & 1006 & 4558 \\
\hline
\end{tabular}

*En esta columna se recogen los resultados de la aplicación del modelo de predicción constant share a los datos reales, y no a la suma de las cifras obtenidas para cada sector.

FUENTE: Elaboración propia.

El modelo de predicción constat-share indica un crecimiento del número empresas cooperativas de 2,8\% entre el año 2015 y 2016. De ese porcentaje, el mayor incremento correspondería al sector de la industria, energía, agua y gestión de residuos con un $0,83 \%$, seguido del sector comercial que aumenta un $0,72 \%$ y los servicios sanitarios, educativos y resto de servicios $(0,58 \%)$.

La tabla 3 indica los diferentes tipos de errores asociados a las predicciones del Modelo de predicción constant share. 


\section{Tabla 3. Diferencias entre los datos obtenidos con la previsión constant-share y los reales. Cálculo de errores}

\begin{tabular}{|l|c|c|c|c|c|c|c|c|c|}
\hline & $\begin{array}{c}\text { Industria, energia, } \\
\text { agua y gestión } \\
\text { de residuos }\end{array}$ & Construcción & Comercio & $\begin{array}{c}\text { Transporte } \\
\text { almacena- } \\
\text { miento }\end{array}$ & Hostelería & $\begin{array}{c}\text { Información } \\
\text { y comunica- } \\
\text { ciones }\end{array}$ & $\begin{array}{c}\text { Banca } \\
y \\
\text { seguros }\end{array}$ & $\begin{array}{c}\text { Servicios } \\
\text { sanitarios, } \\
\text { educativos } \\
\text { y resto servicios }\end{array}$ & Total \\
\hline $\begin{array}{l}\text { SUMA ERRORES } \\
\text { PORCENTAJES DE } \\
\text { ERROR ABSOLUTO }\end{array}$ & 38,29497838 & 60,07056322 & 29,3856546 & 30,96078622 & 46,099771 & 43,85253122 & 50,3044438 & 13,0387104 & 34,0043172 \\
\hline $\begin{array}{l}\text { PORCENTAJE } \\
\text { MEDIO DE } \\
\text { ERROR ABSOLUTO }\end{array}$ & 6,382496397 & 10,01176054 & 4,89760911 & 5,160131037 & 7,6832952 & 7,308755204 & 8,38407396 & 2,1731184 & 5,6673862 \\
\hline Raiz ECM & 10,41004841 & 7,208407195 & 7,58268728 & 1,620628344 & 1,8881457 & 0,529693156 & 0,25171958 & 2,566619058 & 33,4633666 \\
\hline INDICE DE THEIL & 0,035993447 & 0,057104704 & 0,03043197 & 0,034694872 & 0,0463325 & 0,048130691 & 0,0517729 & 0,012800739 & 0,03448538 \\
\hline
\end{tabular}

FUENTE: Elaboración propia.

La tabla 4 recoge los datos resultantes de la aplicación del modelo de predicción constant-shift a la evolución en el número de cooperativas.

\section{Tabla 4. Evolución del número de Cooperativas. Modelo de predicción constant shift}

\begin{tabular}{|c|c|c|c|c|c|c|c|c|c|}
\hline PREVIISOON & $\begin{array}{c}\text { Industria, energía, } \\
\text { agua y gestión } \\
\text { de residuos }\end{array}$ & Construcción & Comercio & $\begin{array}{c}\text { Transporte } \\
\text { y almacena- } \\
\text { miento }\end{array}$ & Hostelería & $\begin{array}{l}\text { Información } \\
\text { y comunica- } \\
\text { ciones }\end{array}$ & $\begin{array}{l}\text { Banca } \\
y \\
\text { seguros }\end{array}$ & $\begin{array}{c}\text { Servicios } \\
\text { sanitarios, } \\
\text { educativos } \\
\text { y resto servicios }\end{array}$ & Total $^{*}$ \\
\hline \multicolumn{10}{|l|}{2008} \\
\hline 2009 & 1759 & 858 & 1484 & 272 & 265 & 70 & 31 & 1057 & 5847 \\
\hline 2010 & 1648 & 730 & 1337 & 238 & 233 & 61 & 27 & 1029 & 5214 \\
\hline 2011 & 1514 & 649 & 1253 & 236 & 211 & 55 & 24 & 1003 & 4933 \\
\hline 2012 & 1419 & 612 & 1200 & 239 & 190 & 56 & 21 & 1008 & 4782 \\
\hline 2013 & 1267 & 543 & 1153 & 223 & 178 & 49 & 23 & 963 & 4374 \\
\hline 2014 & 1255 & 531 & 1173 & 215 & 180 & 48 & 23 & 975 & 4379 \\
\hline 2015 & 1234 & 490 & 1168 & 223 & 175 & 48 & 23 & 999 & 4408 \\
\hline 2016 & 1243 & 491 & 1175 & 223 & 175 & 48 & 23 & 1004 & 4514 \\
\hline
\end{tabular}

*En esta columna se recogen los resultados de la aplicación del modelo de predicción constant shift a los datos reales, y no a la suma de las cifras obtenidas para cada sector.

FUENTE: Elaboración propia. 
El modelo de predicción constant-shift prevé un crecimiento en el número de empresas cooperativas del 2,4\% en el período 2015 a 2016. En el sector industria, energía, agua y gestión de residuos, las empresas cooperativas aumentarían un $0,71 \%$, seguido del comercio $0,61 \%$ y los servicios sanitarios, educativos y resto de servicios $(0,49 \%)$. La tabla 5 recoge los errores asociados a las predicciones efectuadas.

\section{Tabla 5. Diferencias entre los datos obtenidos con el modelo constant-shift y los reales. Cálculo de errores}

\begin{tabular}{|l|c|c|c|c|c|c|c|c|c|}
\hline & $\begin{array}{c}\text { Industria, energía, } \\
\text { agua y gestión } \\
\text { de residuos }\end{array}$ & Construcción & Comercio & $\begin{array}{c}\text { Transporte } \\
\text { y almacena- } \\
\text { miento }\end{array}$ & Hostelería & $\begin{array}{c}\text { Información } \\
y \text { comunica- } \\
\text { ciones }\end{array}$ & $\begin{array}{c}\text { Banca } \\
y \\
\text { seguros }\end{array}$ & $\begin{array}{c}\text { Servicios } \\
\text { sanitarios, } \\
\text { educativos } \\
\text { y resto servicios }\end{array}$ & Total \\
\hline $\begin{array}{l}\text { SUMA ERRORES } \\
\text { PORCENTAJES DE } \\
\text { ERROR ABSOLUTO }\end{array}$ & 36,47104105 & 59,2432561 & 28,2808159 & 30,88910681 & 45,911999 & 43,82087081 & 50,290834 & 13,13977856 & 29,3315267 \\
\hline $\begin{array}{l}\text { PORCENTAJE } \\
\text { MEDIO DE } \\
\text { ERROR ABSOLUTO }\end{array}$ & 6,078506841 & 9,873876017 & 4,71346932 & 5,148184468 & 7,6519998 & 7,303478469 & 8,38180566 & 2,189963093 & 4,88858779 \\
\hline \begin{tabular}{l} 
Raiz ECM \\
\hline INDICE DE THEIL
\end{tabular} & 0,034773454 & 0,056531652 & 0,02945832 & 0,034591072 & 0,0461509 & 0,048093894 & 0,05175551 & 0,012524102 & 0,03065003 \\
\hline
\end{tabular}

FUENTE: Elaboración propia.

Del análisis de los errores de las tablas 3 y 5 se puede concluir que las predicciones realizadas por el modelo de predicción constant shift presentan menores errores que las realizadas por el modelo de predicción constant share para todos los sectores analizados.

\section{8.- Conclusiones}

La reciente crisis económica y su relación con el aumento de los desequilibrios territoriales y sociales evidencian la necesidad de realizar investigaciones que pongan de manifiesto la relación entre las diferentes estrategias desarrolladas por las empresas y su grado de supervivencia. En este artículo se ha analizado, para la comunidad autónoma de Andalucía, el comportamiento de las cooperativas durante la crisis frente al de otro tipo de empresas. 
Del análisis realizado a partir de la información suministrada por el directorio de empresas y establecimientos con actividad económica en Andalucía, se pone de manifiesto que el impacto negativo sobre el empleo en las cooperativas situadas en el territorio andaluz durante la actual crisis económica ha sido menor que el experimentado por el del resto de empresas. Así, desde 2008 a 2014, el porcentaje de disminución del empleo cooperativista fue siete puntos porcentuales menor que el experimentado por otro tipo de empresas.

Los resultados de este trabajo ponen de manifiesto la capacidad de las cooperativas para crear y mantener el empleo, sobre todo, en etapas como la actual de recesión económica. Estas cifras son el resultado de una peculiar forma de entender la gestión empresarial en la cual, por un lado, se prima el papel del trabajo sobre el del capital y, por otro lado, se trabaja en la mejora del grado de proximidad a los clientes, lo que les permite adaptarse de una manera más ágil a los cambios que se producen en el territorio donde ejercen sus actividades.

Sin embargo, al desagregar este análisis territorial a nivel provincial, los resultados obtenidos indican que ese comportamiento no ha sido homogéneo entre las distintas provincias analizadas. Así, las provincias de Almería y Jaén mantienen un porcentaje de empleados en cooperativas sobre el empleo total de empresas mayor al resto de provincias durante todo el período estudiado.

La aplicación del análisis shift-share aplicado a la evolución del empleo en las cooperativas a nivel provincial, corrobora los resultados estadísticos obtenidos a nivel regional. Las provincias de Cádiz, Córdoba y Granada presentan cambios estructurales y cambios diferenciales negativos. Málaga, Jaén y Sevilla tienen cambios estructurales negativos y cambios diferenciales positivos.

Por su parte, los modelos de previsión ofrecen como resultado el mantenimiento de las diferencias provinciales en el tiempo, evidenciando que los cambios en la actividad económica para el período estudiado apenas han influido en la cohesión regional en materia de empleo cooperativo.

Esta situación puede perpetuar un modelo territorial de crecimiento polarizado en diversas unidades territoriales que se retroalimente a sí mismo, debido a la capacidad de atracción de empresas y trabajadores hacia las regiones con bajas tasas de paro, en perjuicio de aquellas que no pueden mantener su nivel de crecimiento en torno a la media andaluza.

En todo caso, el análisis realizado debe ser interpretado como una primera aproximación al estudio de las diferencias de empleo en cooperativas a nivel provincial. Una vez constatadas las mismas en este trabajo de investigación, se hace necesario completarlo mediante un análisis de variables geográficas, económicas, sociales, ambientales, demográficas y culturales asociadas a cada territorio. Son estas las que pueden explicar la existencia de diferentes modelos de crecimiento de carácter territorial en cada una de las provincias estudiadas.

Los resultados obtenidos pueden servir a las entidades públicas para incorporar las conclusiones en sus programas de desarrollo cooperativo regional. Por un lado, priorizando estos programas 
debido a la capacidad que han mostrado este tipo de entidades para adaptarse a los cambios del entorno y mantener el empleo en épocas de crisis de una manera más estable que en otro tipo de empresas; y, por otro lado, incluyendo en los mismos análisis socioeconómicos que pongan de manifiesto las potencialidades de cada territorio y que permitan avanzar en la cohesión de las distintas provincias de la Comunidad Autónoma. Estos análisis deberían contemplar la elaboración de indicadores de desarrollo territorial asociados a las cooperativas. En ambos casos, se hace necesaria la definición de un proyecto territorial común que permita, además de la elaboración de diagnósticos de situación, el desarrollo de medidas e indicadores que puedan ser incorporadas en el proceso de cohesión territorial.

La puesta en marcha de estas políticas de desarrollo regional requiere un proceso consensuado de actuaciones entre los agentes territoriales públicos y privados, tales como las corporaciones locales, los representantes económicos, los sindicatos, las asociaciones, las universidades, etc., junto con las administraciones públicas de ámbito superior al local. De la capacidad para asimilar los cambios que se vienen produciendo por parte de todos los agentes territoriales implicados dependerá la capacidad para revitalizar los territorios. Su negativa, así como la asunción de roles tradicionales, pueden generar pérdidas de oportunidades aumentando así las desigualdades territoriales.

\section{0.- Bibliografía}

ALAÑON, A. \& BUENO, J. (2000): Regional growth and regional imbalances: Spain and USA.

ALBURQUERQUE, F. (2003): Teoría y práctica del enfoque del desarrollo local, Instituto de Economía y Geografía, Consejo Superior de Investigaciones Científicas, Madrid.

ALBURQUERQUE, F. (2004): "Desarrollo económico local y descentralización en América Latina", Revista de la CEPAL, 82, 157-171.

BARFF, R.D. \& KNIGHT, P.L. (1998): "Dynamic Shift-Share analysis", Growth and Change, 19-2, 1-10.

BROWN, H.J. (1969): "Shift-Share projections of regional growth: empirical test", Journal of regional science, 9, 1-18.

BECATTINI, G., COSTA, M.T. \& TRULLÉN, J. (Dir. \& Coord.) (2002): Desarrollo local: Teorías y estrategias, Biblioteca Civitas de Economía y Empresa, Madrid.

BENDAVID-VAL, A. (2001): Análisis económico regional y local para profesionales, Instituto de Desarrollo Regional-Fundación Universitaria, Sevilla.

CAPEL, H. (2009): "Las pequeñas ciudades en la urbanización generalizada y ante la crisis global", Investigaciones geográficas, 70, 7-32. 
CARREE, M.A. \& THURIK, A.R. (2003): "The impact of entrepreneurship on economic growth". Handbook of entrepreneurship research, Springer, 437-471.

CASALIS, A. (2008): "El desarrollo territorial, un desafío para la construcción de un nuevo modelo de desarrollo". Trabajo presentado en las II Jornadas Nacionales de Investigadores de las Economías Regionales, Universidad Nacional del Centro de la Provincia de Buenos Aires.

CLEMENTE, J. et al. (2014): "Evolución del comportamiento estratégico de las cooperativas versus empresas capitalistas: Una aproximación descriptiva al efecto crisis", REVESCO, Revista de Estudios Cooperativos, 116, 77-102.

CUADRADO, M. \& CIRUELA, A.M. (2014): "Las sociedades cooperativas y sociedades laborales como motor de desarrollo económico y social: Análisis de su impacto socioeconómico en la región de Andalucia", REVESCO, Revista de Estudios Cooperativos, 115, 57-100.

DE PAZ, M.A. (2008): "Globalización y gobernanza. Algo más que la tradicional dicotomía estado-mercado", Revista de Economía Mundial, 18, 327-339.

DÍAZ, M. \& MARCUELLO, C. (2010): "Impacto económico de las cooperativas. La generación de empleo en las sociedades cooperativas y su relación con el PIB", CIRIEC-España, Revista de Economía Pública, Social y Cooperativa, 67, 23-44.

ENCINA, B., CALATAYUD, E. \& GARCÍA, G. (2011): "Las cooperativas hortofrutícolas frente a la crisis. La necesaria apuesta por la competitividad. Aspectos económico-financieros", CIRIEC-España, Revista de Economía Pública, Social y Cooperativa, 72, 125-156.

FAJARDO, G. (2009): "La economía social en las leyes", CIRIEC-España, Revista de Economía Pública, Social y Cooperativa, 66, 5-35.

FERNÁNDEZ, A., PAREJO, J.A. \& RODRÍGUEZ, L. (2011): Política económica, $4^{a}$ edición revisada y actualizada, McGraw-Hill, Madrid.

FLORES, M. (2007): "La identidad cultural del territorio como base de una estrategia de desarrollo sostenible", Revista Observatorio de Políticas Públicas Opera, 7, 35-54.

FUENTES, P.D. \& MAINAR, A. (2015): "Impacto económico y en el empleo de la Economía Social en España: Un análisis multisectorial", CIRIEC-España, Revista de Economía Pública, Social y Cooperativa, 83, 63-81.

GARCÍA, J.M. \& MELIÁN, A. (2003): "Evaluación del potencial competitivo de una actividad económica en un territorio desde la perspectiva basada en los recursos y capacidades", Revista Europea de Dirección y Economía de la Empresa, 12, 101-118.

GRÁVALOS, M.A. \& POMEREZ, I. (2001): "Cooperativas, desempleo y efecto refugio", REVESCO, Revista de Estudios Cooperativos, 74, 69-84.

INSTITUTO DE ESTADÍSTICA DE ANDALUCÍA (2008-2014): Directorio de Empresas y Establecimientos con Actividad Económica en Andalucía. 
JAMES, F. \& HUGHES, J. (1973): "A test of shift and share analysis as a predictive device", Journal of regional science, 3, 213-231.

LABARCA, N. (2007): "Consideraciones teóricas de la competitividad empresarial", Omnia, 13(2), 158184.

LÓPEZ, A.J. \& MAYOR, M. (2002): "The evolution of the employment in the European Union. A stochastic shift and share approach", Proceedings of the European Regional Science Association, ERSA 2002, Dortmund.

MAYOR, M., LÓPEZ, A.J. \& PÉREZ, R. (2005): "Escenarios de empleo regional. Una propuesta basada en análisis shift-share", Estudios de economía aplicada, 23-3, 863-887.

MARTÍN-GUZMAN, P. \& MARTíN, J. (1987): Curso básico de estadística económica, Editorial AC, Madrid.

MELIÁN, A. \& CAMPOS, V. (2010): "Emprendedurismo y economía social como mecanismos de inserción sociolaboral en tiempos de crisis", REVESCO, Revista de Estudios Cooperativos, 100, 43-67.

MONZÓN, J.L. (1991): "Análisis del empleo en la economía social", Revista de economía y sociología del trabajo, 12, 17-24.

MONZÓN, J.L. (2006): "Economía Social y conceptos afines: fronteras borrosas y ambigüedades conceptuales del Tercer Sector", CIRIEC-España, Revista de Economía Pública, Social y Cooperativa, 56, 9-24.

NAVARRO, A.M. \& CLIMENT, V.C. (2010): "Emprendedurismo y economía social como mecanismos de inserción sociolaboral en tiempos de crisis", REVESCO, Revista de Estudios Cooperativos, 100, 43-67.

PATUELLI, R., REGGIANI, A., NIJKAMP, P. \& BLIEN, U. (2006): "New neural network methods for forecasting regional employment: an analysis of German labour markets", Spatial Economic Analysis, 1(1), 7-30.

PÉREZ, J.E. \& PIZARRO, J.E. (2012): "La dimensión territorial en la programación comunitaria y el nuevo marco de políticas públicas: desarrollo rural territorial, reforma de la PAC y nuevo LEADER", Anales de Geografía de la Universidad Complutense, 32(2), 227-243.

PÉREZ, M. \& VALIENTE, L. (2015): "Impacto territorial del autoempleo en la economía social en España, CIRIEC-España, Revista de Economía Pública, Social y Cooperativa, 83, 83-114.

PITA, M.F. \& PEDREGAL, B. (2015): "La medición de la cohesión territorial a escala regional. Propuesta metodológica y aplicación a Andalucía", Boletín de la Asociación de Geógrafos Españoles, 68, 31-55.

RAMAJO, J. \& MÁRQUEZ, M.A. (2008): "Componentes espaciales en el modelo Shift-Share. Una aplicación al caso de las regiones peninsulares españolas", Estadística española, 50, 247-272. 
RAMOS, E. \& GARRIDO, D. (Coords.) (2011): Desarrollo rural territorial: Metodología y aplicación para el estudio de casos, Ministerio de Medio Ambiente y Medio Rural y Marino, Madrid.

RAMOS, F.S. \& BAYTER, L.O. (2012): "Emprendimiento y Economía Social, oportunidades y efectos en una sociedad en transformación", CIRIEC-España, Revista de Economía Pública, Social y Cooperativa, 75, 129-143.

RODRÍGUEZ, J.C. (2009): "Los procesos de desarrollo local desde una perspectiva europea: génesis y transformación", Semestre Económico, 12, 37-55.

SALINAS, F. \& OSORIO, L. (2012): "Emprendimiento y Economía social, oportunidades y efectos en una sociedad en transformación", CIRIEC-España, Revista de Economía Pública, Social y Cooperativa, 75, 129-151.

SERVER IZQUIERDO, R.J. \& VICEDO, J.C. (2009): "La Responsabilidad Social Empresarial en un contexto de crisis. Repercusión en las Sociedades Cooperativas", CIRIEC-España, Revista de Economía Pública, Social y Cooperativa, 65, 7-31.

SOBRINO, J. (2005): "Competitividad territorial: ámbitos e indicadores de análisis", Economía, sociedad y territorio, 5(1), 123-183.

TARAZONA, P. \& ALBORS, J. (2005): "La economía social y el desarrollo local", Revista Noticias del CIDEC, 45, 70-75.

TIMMER, M.P. \& SZIRMAI, A. (2000): "Productivity growth in Asian manufacturing: the structural bonus hypothesis examined", Structural change and economic dynamics, 11(4), 371-392.

VÁZQUEZ, A. (2009): "Una salida territorial a la crisis: Lecciones de la experiencia latinoamericana", EURE (Santiago), 35(105), 5-22.

VILLAVERDE, J. (1996): "Impacto de la producción y la productividad sobre el empleo: Una aplicación del análisis Shift-Share a las regiones españolas", Papeles de economía española, 67, 202-221. 
